\title{
Una aproximación a la definición de Patrones a partir de Buenas Prácticas para el desarrollo de sistemas de Gobierno Electrónico
}

\section{An Approach to the Patterns definition from Good Practices for the Electronic Government systems development}

Presentación: 11/10/2019

\section{Doctorando:}

\section{Oscar Carlos Medina}

Universidad Tecnológica Nacional - Facultad Regional Córdoba

omedina@frc.utn.edu.ar

\section{Director/es:}

\section{Mario Alberto Groppo Marcelo Martín Marciszack}

\section{Resumen \\ La propuesta de la presente Tesis de Doctorado es elaborar un modelo de análisis para definir patrones de Buenas Prácticas de e-Gobierno que se puedan aplicar en el Modelo Conceptual de los sistemas del sector público con el fin de facilitar su construcción con mayor nivel de calidad. La dimensión que se intenta optimizar con esta propuesta es el nivel de calidad de sistemas de Gobierno Electrónico de Argentina en particular, y de Iberoamérica en general, al no haberse identificado un modelo de solución exitoso para tomar como punto de partida. Se exponen en este artículo los Objetivos y Metodología de esta investigación, como así también los resultados obtenidos a la fecha y que fueron difundidos en publicaciones científicas y transferencias acreditadas formalmente.}

Palabras claves: Patrones, e-Gob, Buenas Prácticas, Modelado Conceptual, Gobierno Electrónico.

\begin{abstract}
The proposal of this PhD Thesis is to develop an analysis model to define e-Government Good Practices patterns that can be applied in the Conceptual Model of the systems of the public sector in order to facilitate its construction with greater quality level. The dimension that is tried to optimize with this proposal is the Electronic Government systems quality level of Argentina in particular, and of Ibero-America in general, since a successful solution model has not been identified to take as a starting point. The Objectives and Methodology of this research are presented in this paper, as well the results obtained to date, which were diffused in scientific publications and formally accredited transfers.
\end{abstract}

Keywords: Patterns, e-Gov, Good Practices, Conceptual Modeling, Electronic Government.

\section{Introducción}

De la misma forma que algunos sistemas de información basan su diseño en un conjunto de mejores prácticas de negocio para resolver problemáticas bien definidas, como por ejemplo los de ERP (acrónimo en inglés de Planeamiento de Recursos Empresariales), sería conveniente que existieran un conjunto de Buenas Prácticas de Gobierno Electrónico, las cuales pudieran referenciarse y ser utilizadas en el Modelado Conceptual del software e-Gob.

El estudio del Modelado Conceptual de sistemas de información es abordado desde el enfoque de la Ingeniería de Software y en la actualidad se continúa buscando la mejor forma de resolverlo tal como se evidencia en [1], [2] y [3]. 
Una alternativa posible es emplear el concepto de patrones en recomendaciones de prácticas como soluciones adecuadas al contexto de los procesos de negocio gubernamentales, por ejemplo trabajando con distintos tipos de patrones de análisis y diseño. El concepto de patrón es el formulado por Christopher Alexander quien lo define como "una regla de tres partes, que expresa una relación entre un cierto contexto, un problema y una solución" [4]. Se propone construir con ellos un Modelo Conceptual que se adapte al nuevo contexto aprovechando la propiedad de reusabilidad de la solución que ofrecen los patrones.

Para lograr este cometido, la investigación subyacente de la Tesis de Doctoral plantea los siguientes objetivos:

a) Objetivo general

Definir patrones a partir de Buenas Prácticas que puedan incorporarse en el Modelo Conceptual de sistemas de Gobierno Electrónico de Argentina, en el marco de un modelo iberoamericano de software público.

b) Objetivos específicos

i) Caracterizar el conjunto de Buenas Prácticas de Gobierno Electrónico más representativas en la actualidad y en un contexto iberoamericano.

ii) Identificar los tipos de patrones de software existentes que permitan definir Buenas Prácticas de eGobierno y describir sus características básicas.

iii) Describir un modelo de análisis que permita definir patrones de Buenas Prácticas de e-Gobierno.

iv) Proponer un método de selección y aplicación de patrones de Buenas Prácticas en el Modelo Conceptual de sistemas de Gobierno Electrónico.

v) Evaluar la aplicación de estos patrones de Buenas Prácticas en sistemas de Gobierno Electrónico utilizando un modelo de calidad de software específico.

El diseño metodológico de este trabajo tiene un enfoque cualitativo con estudios exploratorios de Buenas Prácticas de Gobierno Electrónico y de patrones de software dentro del marco teórico de la Ingeniería de Software. Se propone realizar un análisis descriptivo de los patrones que pueden aplicarse en el Modelo Conceptual de software público iberoamericano. Como resultado de la investigación se obtendrá:

a) Un modelo de análisis que permita definir Buenas Prácticas de e-Gobierno utilizando patrones como herramienta

b) Un método de selección y aplicación de estos patrones a un nuevo sistema e-Gob.

c) La comprobación de manera experimental que con este nuevo modelo de análisis se optimizó el nivel de calidad del software de acuerdo a un modelo de medición y evaluación específico para sistemas de Gobierno Electrónico utilizando un procedimiento multicriterio AHP (Analytic Hierarchy Process).

\section{Resultados}

Los resultados obtenidos a la fecha, en función de los objetivos de la investigación, se pueden agrupar en las siguientes áreas de incumbencia:

a) Proyectos de $\mathrm{I}+\mathrm{D}+\mathrm{i}$.

b) Libros.

c) Capítulos de libros.

d) Publicaciones con referato en revistas científicas.

e) Publicaciones en congresos y reuniones científicas.

f) Transferencias acreditadas formalmente.

A continuación se describen brevemente cada uno de estos resultados señalando sus referencias en caso que se desee profundizar en el contenido.

Proyectos de I+D+i. La Tesis Doctoral se radicó inicialmente en el proyecto de investigación homologado PID 3604 "Implementación de patrones en la validación de Modelos Conceptuales" que finalizó el 31/12/2018. Pero antes se conformó un equipo de docentes investigadores y becarios que aplicaron y obtuvieron la aprobación de dos nuevos proyectos de investigación con financiamiento y con foco en el tema central de la Tesis. En primer lugar el PID UTN4853 "Un Modelo de Análisis para aplicación de Patrones de buenas prácticas en el Modelado Conceptual de Gobierno Electrónico" dirigido por el Prof. Dr. Mario Alberto Groppo y codirigido por el Prof. Ing. Calixto Maldonado con un plan de desarrollo desde el 01/01/2018 hasta el 31/12/2020. También se obtuvo la aprobación, Resolución $N^{\circ} 79 / 2018$, de un financiamiento del programa G.R.F.T., Grupos de Reciente Formación con Tutores, del Ministerio de Ciencia y Tecnología de la Provincia de Córdoba, para llevar a cabo el proyecto de investigación intermedio "Propuesta para la definición de Patrones a partir de Buenas Prácticas para el desarrollo de sistemas de Gobierno 
Electrónico" dirigido por el doctorando y tutorado por el Prof. Dr. Mario Alberto Groppo desde el 01/01/ 2019 hasta el 31/07/2020. En ambos proyectos se desarrollan actividades de investigación concomitantes con los objetivos específicos de la Tesis Doctoral.

Libros. El doctorando es coautor del libro "Patrones en la construcción del Modelo Conceptual para sistemas de información" [5] publicado con financiamiento del programa PRO.TR.I., Proyectos de Transferencia de Resultados de Investigación y Comunicación Pública en la Ciencia, del Ministerio de Ciencia y Tecnología de la Provincia de Córdoba, Resolución N 106/2017. La primera edición de diciembre de 2018 consta de 300 ejemplares en papel pero además se distribuye en forma gratuita la versión electrónica desde el sitio web del Centro de Investigación CIDS de U.T.N. F.R.C.: http://www.cids.frc.utn.edu.ar/. En esta publicación se formalizó una propuesta para la definición de un Modelo Conceptual en el desarrollo de Sistemas de Información, que tiene como punto de partida la utilización de Patrones de Procesos del modelo de negocios. En ese marco, se identificaron los patrones de procesos que permiten definir Buenas Prácticas de e-Gobierno, se describieron sus características básicas y se ejemplificó con un caso práctico de aplicación en un Organismo Público. El doctorando expuso recientemente la publicación en formato poster en COPUCI 2019 [6].

Capítulos de libros. El mencionado caso práctico fue desarrollado más en detalle y publicado como capítulo del libro "Trends and Applications in Software Engineering" [7]. En este trabajo se expone la aplicación de patrones de procesos y de objetivos en el modelado de un software móvil denominado "Ubicuo" que está implementado, entre otros clientes, en EPEC, la empresa pública de energía de Córdoba. Nuevamente en el mismo libro, se va a publicar en la edición del presente año, otro trabajo donde el doctorando es coautor [8]. En este artículo se pone a consideración el empleo de patrones de objetivos en el Modelado de Negocios de un sistema de Educación Pública, también para la Provincia de Córdoba.

Publicaciones con referato en revistas científicas. Se publicó en la revista científica de U.TN. un artículo [9] que introduce en la temática de los sistemas de Gobierno Electrónico de Argentina, en un marco referencial iberoamericano, a partir de la enunciación de los conceptos básicos de E-Gobierno y Modelado Conceptual desde el punto de vista de la Ingeniería de Software hasta el relevamiento de su situación actual, a los fines de identificar y caracterizar la incorporación de buenas prácticas en el Modelo Conceptual de este software.

Publicaciones en congresos y reuniones científicas. Se puso a consideración de la comunidad científica nacional e internacional la propuesta de análisis de la Tesis Doctoral que consiste en caracterizar patrones de Buenas Prácticas de E-Gobierno que se puedan utilizar en el Modelo Conceptual de los sitios web del sector público para que facilite su construcción con mayor nivel de calidad. Se expuso y publicó en Argentina en las jornadas WICC 2018 [10] y en España en CISTI 2018 [11]. También se presentó y publicó en CONAIISI 2018 un tercer trabajo [12] sobre la incorporación de patrones de procesos y objetivos en el modelado del sistema de gestión de consultas de ciudadanos de la Caja de Jubilaciones, Pensiones y Retiros de Córdoba. Los patrones publicados en [7], [8] y [12] forman parte de un catálogo de patrones para poner a prueba, en la fase de experimentación de la Tesis Doctoral, método de selección y aplicación de estos patrones a un nuevo sistema e-Gob.

Transferencias acreditadas formalmente. Dentro de las transferencias de resultados del proyecto de investigación PID 3604 "Implementación de patrones en la validación de Modelos Conceptuales", se formalizó con dos instrumentos las actividades de formación y trabajo en conjunto con la Caja de Jubilaciones, Pensiones y Retiros de Córdoba. Por una parte se firmó un convenio de transferencia en el año 2017 que se iniciaba con el dictado del taller "Utilizando patrones para comprender procesos de negocio" en las oficinas del Organismo Público. A posteriori se realizaron actividades de modelado utilizando patrones en un módulo del sistema informático de la Entidad y se elaboraron artículos en conjunto, que fueron compilados y presentados en un PDTS, Proyecto de Desarrollo Tecnológico y Social, aprobado en el corriente año por la Secretaría de Ciencia, Tecnología y Posgrado de U.T.N. para ser incorporado al Banco de Proyectos de Desarrollo Tecnológico y Social (BPDTS) de la Secretaría de Gobierno de Ciencia, Tecnología e Innovación Productiva de la Nación. Además, el artículo [11] fue presentado en "Vincular Córdoba 2019" en formato de poster en U.T.N. - F.R.C., y se dio una breve disertación denominada PDO sobre "Transferencia de resultados de Investigación a una Carrera Universitaria" junto con el Co-Director de la Tesis Doctoral [13].

Finalmente, se hace una breve reseña de las actividades en ejecución y los próximos pasos. Se presentaron artículos sobre casos de estudio del empleo de patrones de arquitectura y patrones de escenario en sistemas de Gobierno Electrónico. También está en desarrollo en un lenguaje de código abierto un software denominado "PatCat - Pattern Catalogue", que permite a modo de prueba de concepto evaluar un método de selección de patrones de un catálogo predeterminado a partir de la identificación de un problema ya resuelto anteriormente en el ámbito de E-Gobierno. La próxima actividad de la investigación es el diseño, ejecución y análisis de la fase experimental de la Tesis Doctoral. El 
objetivo es evaluar si la propuesta de análisis, incorporando patrones al Modelo Conceptual inicial, optimiza el nivel de calidad del software de acuerdo a un modelo de medición y evaluación específico para sistemas de Gobierno Electrónico, para lo cual se seleccionó al Modelo MoQGEL [14]. Se prevé instrumentar el experimento empleando métodos de Ingeniería de Software basada en evidencias como los descriptos en [15].

\section{Referencias}

[1] Pohl, K. \& Rupp, C. (2015). Requirements Engineering Fundamentals: A Study Guide for the Certified Professional for Requirements Engineering Exam - Foundation Level - IREB compliant. 2nd Edition, International Requirements Engineering Board.

[2] Sommerville, I. (2011). Ingeniería de Software. 9a Edición en español”, Pearson.

[3] Wiegers, K. \& Beaatty, J. (2013). Software Requirements (3rd Edition) (Developer Best Practices). Microsoft.

[4] Alexander, C. (1979). The Timeless Way of Building. Oxford University Press - New York.

[5] Marciszack, M.M., Moreno, J.C., Sánchez, C.E., Medina, O.C., Delgado, A.F., Castro, C.S. (2018). Patrones en la construcción del Modelo Conceptual para sistemas de información. Editorial edUTecNe, U.T.N.

[6] COPUCI 2019 - VII Congreso Internacional de Comunicación Pública de la Ciencia y la Tecnología. Extraído el 11/10/2019, de https://copuci.wordpress.com.

[7] Medina, O.C, Pérez Cota, M., Marciszack, M.M., Martin, S.M., Pérez, N., Dean, D.D. (2018). Conceptual Modelling of a Mobile App for Occupational Safety Using Process and Objectives Patterns. Publicado en "Trends and Applications in Software Engineering" págs. 186-195, Springer.

[8] Meloni, B.E., Pérez Cota, M., Medina, O.C., Marciszack, M.M. (2019). Objectives Patterns Applied to the Business Model of a Public Education System. A publicarse en "Trends and Applications in Software Engineering", Springer.

[9] Medina, O.C, Marciszack, M.M., Groppo, M.A. (2018). Aproximación descriptiva a las Buenas Prácticas de Gobierno Electrónico y a su incorporación en el Modelado Conceptual de Sitios Web Públicos de Argentina. Revista

“Tecnología y Ciencia” en línea, N 31, Artículo 107, Universidad Tecnológica Nacional, Argentina.

[10] Medina, O.C, Marciszack, M.M., Groppo, M.A. (2018). Un Modelo de Análisis para aplicación de patrones de Buenas Prácticas en el Modelado Conceptual de Gobierno Electrónico. Publicado en actas de WICC 2018, Red UNCI y UNNE Universidad Nacional del Nordeste.

[11] Medina, O.C, Marciszack, M.M., Groppo, M.A. (2018). Proposal for the patterns definition based on good practices for the electronic government systems development. Publicado en actas de CISTI 2018 - 13th Iberian Conference on Information Systems and Technologies.

[12] Medina, O.C, Cánepa, P.A., Gruppo, M.O., Groppo, M.A. (2018). Un caso de estudio de patrones de Gobierno Electrónico para gestión de consultas de ciudadanos. Publicado en actas de CONAIISI 2018, Red RIISIC, CONFEDI y Universidad CAECE. 
[13] Vincular Córdoba 2019 - Jornadas de articulación para la Innovación 5 Edición. Extraído el 11/10/2019, de https://vincularcordoba.com.

[14] Sá, F.A., \& Rocha, A. (2017). Qualidade do Governo Eletrónico. Modelo MoQGEL. Ed. Sílabo.

[15] Genero Bocco, M., Cruz-Lemus, J.A., Piattini Velthuis, M.G. (2014). Métodos de investigación en ingeniería del software. Ed. Ra-Ma. 